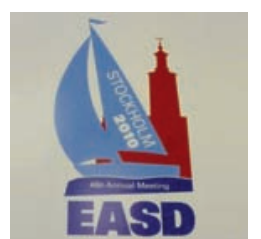

gen Kindern verändert ist, die Autoantikörper haben. Da die Darmbakterien die Zusammensetzung der Aminosäuren und Fette im Blut beeinflussen, könnte es sein, dass Veränderungen in der Zusammensetzung durch einen veränderten Lebensstil zur erhöhten Rate von Typ-1-Diabetes beitragen, so der finnische Wissenschaftler. Und er kann auch einen historischen Befund präsentieren. In einer
Studie ging die Zunahme der Inzidenz des Typ-1Diabetes mit der Zeit parallel mit der Entwicklung des Bruttosozialproduktes. Welche Präventionsoptionen ergäben sich, wenn weitere Forschungen die Hypothesen bestätigen? Vakzine gegen die triggernden Viren und Vermeiden der boosternden Faktoren, so die Antwort.

Dieter Rödder

\title{
Typ-1-Diabetes bei Kindern
}

Vorkommen: Etwa 22000 Kinder und Jugendliche in Deutschland haben Diabetes, über 99\% davon Typ-1-Diabetes. Auch in Deutschland steigt die Inzidenz, und zwar um rund 3,5\% pro Jahr, derzeit sind es 17 Neuerkrankungen pro 100000 Einwohner jährlich. Die Ursachenforschung ist noch lückenhaft. Es gibt Hinweise, dass Stillen über fünf Monate das Risiko reduziert und das Zufüttern von Kuhmilch das Risiko erhöht.

Forschung: In der BABYDIAB-Studie werden Säuglinge von Eltern mit Typ-1-Diabetes betreut. Kinder, die schon in den ersten vier Lebensmonaten Beikost erhielten, hatten ein vierfaches Risiko für die Entwicklung von Autoantikörpern gegen PankreasInselzellen und Typ-1-Diabetes. Die Beikost enthielt oft Getreide, also Gluten. In der Studie werden 150 Säuglinge mit hohem Diabetesrisiko (Risikogene plus ein erstgradiger Angehöriger mit Typ-1-Diabetes) sechs oder zwölf Monate lang glutenfrei ernährt.

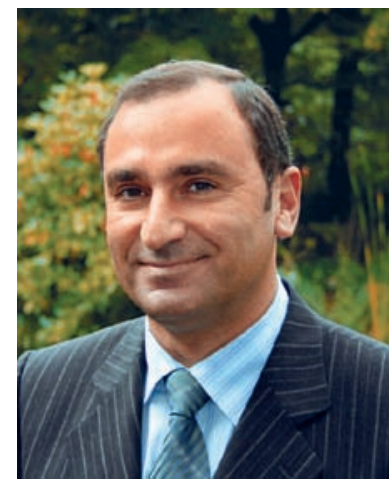

Prof. Dr. med Dan Ziegler, Düsseldorf, Träger des Castelli Pedroli Preises und Schriftleiter von Info Diabetologie.

\section{Professor Dan Ziegler erhält Castelli-Pedroli-Preis}

\section{Polyneuropathie ist nicht nur Spätkomplikation eines Diabetes}

Für seine umfangreichen Forschungen zur diabetischen Neuropathie hat der Diabetologe Professor Dan Ziegler von der Universität Düsseldorf den Castelli-PedroliPreis erhalten. Der Preis ist verknüpft mit der Ehre, beim EASD-Kongress die Camillo-Golgi-Lecture zu halten.

Ziegler hat in seinem Vortrag darauf hingewiesen, dass diabetische Polyneuropathie (DPN) nicht nur eine Spätkomplikation des Diabetes ist, sondern sich bereits sehr früh in der Erkrankung manifestieren kann. Mithilfe von ausgeklügelten objektiven Techniken zur Entdeckung von Nervenschäden, konnte er mit seiner Arbeitsgruppe zeigen, dass bereits bei der Diagnose von Typ-1- und Typ-2-Diabetes viele Störungen der Nervenfunktion nachweisbar sind, z.B. an Unterschenkeln, im Herz und im Magen.

Einen Beleg dafür, wie häufig Neuropathien in den verschiedenen Stadien sind, liefern die Daten der bevölkerungsbasierten Studie MONICA/KORA in der Region Augsburg. Von den Diabetikern unter den Studienteilnehmern hatten $28 \%$ eine DPN. Die Prävalenz einer schmerzhaften Neuropathie war damit zwei- bis dreimal höher bei Studienteilnehmern mit gestörter Glukosetoleranz, bei der sie $9 \%$ betrug, und bei Diabetes, bei der die Prävalenz
$13 \%$ betrug, im Vergleich zu solchen mit erhöhter Nüchternglukose. Bei ihnen lag die Prävalenz bei 4\%. Die hauptsächlichen Risikofaktoren für eine DPN sind, abgesehen von Diabetes das Alter, Adipositas, PAVK und niedriggradige systemische Entzündung. Dass oxidativer Stress bei der Pathogenese der DPN eine Bedeutung hat, ist bereits aus Tiermodellen bekannt. Ziegler konnte nachweisen, dass Biomarker für oxidativen Stress und verminderte antioxidative Wirkung wie das SuperoxidAnion und Vitamin E die Entwicklung und Progression der DPN vorhersagen können. Solche Substanzen könnten als Biomarker für ein erhöhtes Neuropathierisiko in der Praxis dienen.

Dass normale Blut-Glukose-Spiegel ab der Diagnose die Entwicklung einer diabetischen Polyneuropathie komplett verhindern können, hat Ziegler in einer prospektiven Beobachtungsstudie über 24 Jahre mit Typ-1-Diabetikern nachgewiesen, während von den schlecht eingestellten Diabetikern zwei Drittel die Erkrankung bekommen.

Das Ziel ist, eine Dysfunktion der Nerven früh zu erkennen, Patienten zu identifizieren, die ein erhöhtes Risiko für eine Progression haben, und diese zu verhindern.

rö 